\title{
Detection and characterization of human rotavirus in hospitalized patients in the cities of Ponta Grossa, Londrina and Assai - Pr, Brazil
}

\begin{abstract}
Acute diarrheal disease is still one of the major public health problems worldwide. Rotaviruses (RV) are the most important viral etiologic agents and children under five years of age are the target population. Objective: To investigate the rate of RV infection in hospitalized patients due to acute diarrhea in the cities of Ponta Grossa, Londrina and Assai - Paraná. Methods: Latex agglutination (LA); immunochromatography (ICG); polyacrylamide gel electrophoresis (PAGE) and negative staining electron microscopy (ME) tests were used to detect the virus. For the genotyping, RT-PCR and RT-PCR-ELISA were used, respectively, for NSP4 and VP4/VP7. Result: Out of 124 samples there were 69 positive stool samples for RV, for at least one of the used tests, 67 of them being RV group A (RV-A). Overall, most of the RV positive stool samples came from children under thirteen years of age. However, 12 positive cases occurred in patients aged 13 years or above, including an 81-year old patient. Conclusion: The data showed similar electropherotypes and genotypes G, P and NSP4 of the inland wild circulating strains of RV.
\end{abstract}

Keywords: rotavirus; determination; hospitalized patients; genotyping.

[Braz J Infect Dis 2010;14(6):553-557] @Elsevier Editora Ltda.

\section{INTRODUCTION}

Acute diarrheal disease is still one of the major public health problems worldwide. Rotaviruses (RV) are the most important etiologic agents and children under five years of age are the preferred target population. Moreover, a variety of infant animals are equally affected by species-specific virus strains. Worldwide, RV cause approximately 112 millions of domestic episodes of diarrhea, 25 millions of clinic visits, 2 millions of hospitalizations and about 611,000 deaths of children under five years of age, annually. ${ }^{1}$

$\mathrm{RV}$ are icosahedric virions, non-enveloped, and present a triple concentric layers of proteins. ${ }^{2,3}$ The inner layer is formed by virus protein 2 (VP2) that involves the genome, the VP1 (RNA polymerase dependent of RNA) e a VP3 (guanilyltransferase and methylase). The intermediate layer is made up of VP6 associated with VP2 and confers the structure the so-called double-layered particles (DLP). The outer layer is constituted by trimeric structures of VP7 glycoprotein and the dimeric spikes of VP4 forming the triple-layered particles (TLP), the infectious form of the virus. Virus genome is represented by 11 segments of double-stranded RNA that encode, respectively, six structural proteins (VP1-VP4, VP6 and VP7) and six nonstructural proteins (NSP1-NSP6).

Based on antigenic specificity of VP6, RV are classified into seven groups (A-G), prevailing the infections by group A strains (RV-A). ${ }^{2}$ Moreover, VP6 epitopes allow the differentiation of group A strains into sub-groups (SG-I, SG-II, SG-I/II, and non-SG-I/II), prevailing SG-II in human infections. ${ }^{4}$ These strains are molecularly distinguished into genogroup I (SG-I) and genogroup II (SG-II, SG-I/II, and SG non-I,non II). ${ }^{5} \mathrm{G}$ and P genotyping is attributed, respectively, to VP7 and VP4. ${ }^{2}$ Twenty-three $\mathrm{G}$ and $31 \mathrm{P}$ genotypes have been described. ${ }^{6,7}$ Due to the importance of NSP4 protein in the virion morphogenesis, replication and in the pathogeny of the infection this protein was also defined molecularly into six genotypes (A-F). ${ }^{4}$

Genotypes G1-G4 and G9 combined with $\mathrm{P}[8]$ and $\mathrm{P}[4]$ are the most prevalent worldwide (approximately 90\%). ${ }^{9,10}$ Concerning NSP4 genotyping, B type has been shown to be the most common in the world. ${ }^{11,12}$

A more complex though complete classification of group A RV has been proposed based on the molecular analysis of all eleven genome segments. ${ }^{8}$
Authors

Carlos M Nozawa ${ }^{1}$

Gustavo Franthesco Kerntopf ${ }^{2}$

Érika da Silva Czernisz

Daniele Albuquerque ${ }^{3}$

Priscila Romanin ${ }^{4}$

José Felipe Eliseu Freitas ${ }^{4}$

Norma Santos 5

Fabrício José Benati ${ }^{6}$

Eduardo Pietruchinski

Rosa Elisa Carvalho Linhares

${ }^{1} \mathrm{PhD}$ in Sciences (Virology)

Universidade Estadual de

Londrina, Londrina, Pr.

${ }^{2}$ Master's Degree in

Microbiology/Universidade

Estadual de Londrina,

Londrina, Pr.

${ }^{3}$ Bacheloring in Biological

Sciences/ Universidade Estadual de Londrina, Londrina, Pr.

${ }^{4}$ Secondary School - Fellowship

of IC Jr/ Universidade Estadual

de Londrina, Londrina, Pr.

${ }^{5} \mathrm{PhD}$ in Sciences (Microbiology)

Universidade Federal do Rio de

Janeiro, Rio de Janeiro, RJ

${ }^{6}$ Master's Degree in

Microbiology/Universidade

Federal do Rio de Janeiro, Rio

de Janeiro, RJ.

${ }^{7}$ Master's Degree in

Microbiology/Centro de

Ensino Superior de Campos

Gerais, Ponta Grossa, Pr.

${ }^{8} \mathrm{PhD}$ in Sciences (Microbiology)/

Universidade Estadual de

Londrina, Londrina, Pr.

Submitted on: 3/2/2010

Approved on: 9/6/2010

Correspondence to:

Universidade Estadual de Londrina

Rodovia Celso Garcia Cid,

(Pr 445) Km 380

Caixa Postal 6001

CEP: 86.051-990 - Londrina

Paraná - Brasil

Phone: +55 (43) 3371-4000

E-mail: cnoz@uel.br

Financial Support: $\mathrm{CNPq}$, Brazilian Ministry of Health, Capes, Fundação Araucária, Proppg/UEL

We declare no conflict of interest. 
By poliacrylamide gel electrophoresis (PAGE) RV are classified into seven electropherotypes (e-type) from A-G, according to the migration pattern of the 11 RNA segments. Moreover, group A strains can be further classified according to the mobility of the segments 10 and 11 into long (L), short (S) and super-short (SS) electropherotypes according to the migration pattern. ${ }^{13,14}$

Presently, two types of RV vaccine are commercially available for human use (Rotarix and RotaTeq) and their safety and efficacy to prevent and/or attenuate severe diarrheal episodes have been proved..$^{15,16}$

Bearing in mind the genetic variability of the virus, either vaccine or host natural immunity to the virus or both may pose a selective pressure that may result in emergence of unusual genotypes. Crossing species barrier is also another possibility for the appearance of mutant strains. In fact, hitherto undescribed genotypes have been found. ${ }^{17}$ These selective events may represent a significant antigenic "shift" or "drift", as has been shown for influenza virus with a real impact in the epidemiology of the disease. Therefore, it is important to monitor wild strains of the virus in order to evaluate all these consequences, and to accompany the evolution of the infection.

In this paper we evaluated circulating human RV strains in three locations of the State of Paraná, Brazil.

\section{MATERIAL AND METHODS}

\section{Feces}

One hundred and twenty four fecal samples were collected from April 2005 to March 2009 from hospitalized patients suffering from acute diarrhea, admitted to private and public hospitals in the cities of Assai (Hospital Climas), Londrina (Hospital Universitário Regional do Norte do Paraná) and Ponta Grossa (Hospital Bom Jesus) - Paraná. The study protocol was approved by the Experimental Ethics Committee of the Universidade Estadual de Londrina, under the ${ }^{\circ}$ 01840268000-07.

\section{Negative staining electron microscopy}

For transmission electron microscopy (EM) raw stool samples were processed by super direct negative staining with $2 \%$ sodium phosphotungstate, $\mathrm{pH} 6.3$, as described elsewhere. ${ }^{18}$

\section{Immunochromatography and latex agglutination tests}

Stool samples were also homogenized at $20 \%(\mathrm{vol} / \mathrm{vol})$ in PBS, pH 7.3, and clarified by centrifugation at $450 \mathrm{xg} / 10 \mathrm{~min}$. Clarified homogenate were submitted to Vikia - Rota Adeno, Biomérieux SA, Fr. and/or Virotect Rota, Omega Diagnostic Ltd., UK., according to the manufacturers recommendations.

\section{Polyacrilamide gel electrophoresis}

Clarified fecal homogenates were further submitted to virus RNA extraction, as described before, ${ }^{19}$ for PAGE.

\section{RT-PCR and PCR-ELISA}

For RT-PCR virus RNA was extracted from stools using the TRIzol method (Invitrogen, Carlsbad, USA) and subjected to reverse transcription followed by PCR..$^{20}$ The cDNA of VP7 or VP4 gene was synthesized by using primers labeled with biotin at their 5' ends. For PCR-ELISA detection of labeled PCR products and identification of the genotypes of positive samples, briefly, 96-well microplates (Nunc-Immuno module, Nunc, Roskilde, Denmark) coated with streptavidin (Roche Diagnostic GmbH, Mannheim, Germany) were used. To each well the biotin-labeled PCR product was distributed (one reaction per genotype) followed by the addition of type-specific digoxigenin-labeled probe. Individual G- or P-type specific probe mixtures (three type-specific probes/genotype/mixture) were distributed onto the plates. This was followed by the addition of anti-digoxigenin peroxidase conjugate (Roche Diagnostic $\mathrm{GmbH}$ ); the substrate (TMB peroxidase substrate system; KPL, Gaithersburg, $\mathrm{MD}$ ), and absorbance read at $450 \mathrm{~nm}$ within $10 \mathrm{~min} .{ }^{21}$ For NSP4 typing, nested-PCR was used and primers are listed in Table 1, including those for VP7 and VP4 typing.

Table 1. VP7, VP4 and NSP4 primers used for amplification and genotyping

\begin{tabular}{|lccc|}
\hline Primers & \multicolumn{1}{c}{ Sequences } & Positions & Genes \\
\hline Beg9 $(+)$ & GGCTTTAAAAGAGAGAATTTCCGTCTGG & $1036-1062$ & VP7 \\
\hline End9 $(-)$ & GGTCACATCATACAATTCTAATCTAAG & $1-28$ & VP7 \\
\hline Con2 $(-)$ & ATTTCGGACCATTTATAACC & $887-868$ & VP4 \\
\hline Con3 $(+)$ & TGGCTTCGCTCATTTATAGACA & $11-32$ & VP4 \\
\hline NSP4-1a $(+)$ & GGCTTTTAAAAGTTCTGTTCCG & $1-12$ & NSP4 \\
\hline NSP4-2b $(-)$ & GGTCACATTAAGACCGTTCC & $750-731$ & NSP4 \\
\hline NSP4-Kun-1a $(+)$ & ATTGATAGTGCGATCGACTGG & $439-460$ & NSP4-A \\
\hline NSP4-Wa-1a $(+)$ & GGCTGGATATAAAGAGCAGG & $286-306$ & NSP4-B \\
\hline NSP4-RRV-1a $(+)$ & AACGATTGGGCTGAAAGGTG & $508-528$ & NSP4-C \\
\hline
\end{tabular}




\section{RESULTS}

From 124 stool samples evaluated 69 were positive for RV (55.6\%), for at least one of the methods used. Amongst these 69 positive samples, 63 (88.4\%) were considered group A (RV-A), as demonstrated by serological methods, being positive either by ICG or LA. Additionally, four strains were also defined as RV-A by electrophoretic pattern by PAGE. Therefore, overwhelmingly, a total of 67 strains out of 69 (97.1\%) were RV-A. Individually, the performance of the methods used accounted for the following results (Table 2): For LA, 66 samples were analyzed and 23 were positive (34.8\%). For ICG, 40 samples were positive out of $64(62.5 \%)$. For PAGE, out of 124 samples 47 were positive (37.9\%), being 26 strains, $55.2 \%$ (26/47), with short pattern electropherotypes and 20,42.5\% (20/47), with long pattern. For EM, 47 samples were tested and 11 were positive (23.4\%). As far as genotyping is concerned, out of the 124 samples, 44 samples (35.4\%) were amplified as following.

Table 2. Rotavirus strains with the respective methods of detection/typing

\begin{tabular}{|c|c|c|c|c|c|c|c|c|}
\hline \multirow[t]{2}{*}{ Strain } & \multirow{2}{*}{$\begin{array}{l}\text { Age } \\
\text { (yrs./ } \\
\text { mos.") }\end{array}$} & \multirow[t]{2}{*}{ LA } & \multirow[t]{2}{*}{ ICG } & \multirow[t]{2}{*}{ E-type ${ }^{a}$} & \multirow[t]{2}{*}{ EM } & \multicolumn{3}{|c|}{ Genotypes $^{b}$} \\
\hline & & & & & & VP7 & VP4 I & NSP4 \\
\hline ASS08/001 & 1 & $\mathrm{ND}^{\mathrm{c}}$ & - & - & + & $\mathrm{NA}^{\mathrm{d}}$ & NA & NA \\
\hline ASS08/003 & 4 & ND & + & $\mathrm{L}$ & + & $\mathrm{NTD}^{\mathrm{e}}$ & NTD & A \\
\hline ASS08/004 & 2 & ND & - & - & + & NA & NA & NA \\
\hline LON08/016 & 2 & + & ND & S & ND & G2 & $\mathrm{P}[4]$ & A \\
\hline LON08/018 & 2 & + & ND & S & ND & G2 & $\mathrm{P}[4]$ & $\mathrm{A}$ \\
\hline LON08/019 & 3 & - & ND & S & ND & NTD & NTD & A \\
\hline LON08/022 & 7 & + & ND & $S$ & + & NTD & NTD & NTD \\
\hline PGR05/001 & 1 & - & ND & - & - & NTD & NTD & B \\
\hline PGR05/002 & 3 & + & ND & $\mathrm{L}$ & ND & G1 & NTD & B \\
\hline PGR05/006 & 3 & + & ND & - & - & NTD & NTD & NTD \\
\hline PGR05/010 & 11 & - & ND & $\mathrm{L}$ & + & G1 & $\mathrm{P}[8]$ & B \\
\hline PGR05/011 & 6 & + & ND & $\mathrm{L}$ & ND & NTD & $\mathrm{P}[8]$ & B \\
\hline PGR05/012 & 1 & + & ND & $\mathrm{L}$ & ND & NTD & NTD & NTD \\
\hline PGR05/013 & 10 & + & ND & - & - & NA & NA & NA \\
\hline PGR05/015 & 1 & + & ND & $\mathrm{L}$ & ND & G1 & $\mathrm{P}[8]$ & B \\
\hline PGR05/016 & 12 & + & ND & $\mathrm{L}$ & ND & G1 & $\mathrm{P}[8]$ & B \\
\hline PGR05/019 & 14 & - & ND & - & - & NTD & NTD & B \\
\hline PGR05/020 & 2 & + & ND & $\mathrm{L}$ & ND & NTD & NTD & B \\
\hline PGR05/021 & 9 & + & ND & - & - & NA & NA & NA \\
\hline PGR05/023 & $4 *$ & + & ND & $\mathrm{L}$ & ND & NTD & NTD & NTD \\
\hline PGR05/024 & $8^{*}$ & - & ND & $\mathrm{L}$ & ND & NTD & NTD & B \\
\hline PGR05/025 & 1 & + & ND & - & ND & NTD & NTD & NTD \\
\hline PGR05/026 & 14 & + & ND & S & ND & NTD & $\mathrm{P}[8]$ & B \\
\hline PGR05/027 & 9* & + & ND & $\mathrm{L}$ & ND & G1 & $\mathrm{P}[8]$ & B \\
\hline PGR05/030 & 1 & - & ND & $\mathrm{L}$ & ND & NTD & NTD & NTD \\
\hline PGR05/031 & 7 & + & ND & $\mathrm{L}$ & ND & NTD & NTD & NTD \\
\hline
\end{tabular}

\begin{tabular}{|c|c|c|c|c|c|c|c|c|}
\hline PGR05/032 & 7 & + & ND & $\mathrm{L}$ & ND & NTD & NTD & NTD \\
\hline PGR05/033 & 4 & + & ND & $\mathrm{L}$ & ND & G1 & $\mathrm{P}[8]$ & B \\
\hline PGR05/034 & 1 & + & ND & - & ND & G1 & NTD & B \\
\hline PGR05/039 & 2 & + & ND & $\mathrm{L}$ & + & G1 & $\mathrm{P}[8]$ & B \\
\hline PGR05/042 & 58 & + & ND & $\mathrm{L}$ & + & G9 & $\mathrm{P}[8]$ & B \\
\hline PGR05/043 & 46 & + & ND & $\mathrm{L}$ & ND & NTD & $\mathrm{P}[8]$ & B \\
\hline PGR05/045 & 22 & + & ND & NTD & ND & NTD & NTD & B \\
\hline PGR05/046 & $8^{*}$ & + & ND & $\mathrm{L}$ & ND & G9 & $\mathrm{P}[8]$ & B \\
\hline PGR05/049 & 3 & + & ND & $\mathrm{L}$ & + & G1 & $\mathrm{P}[8]$ & NTD \\
\hline PGR08/001 & 4 & ND & + & S & ND & NTD & $\mathrm{P}[4]$ & A \\
\hline PGR08/002 & 81 & ND & + & S & ND & G2 & $\mathrm{P}[4]$ & A \\
\hline PGR08/003 & 5 & ND & + & - & - & NA & NA & NA \\
\hline PGR08/004 & 4 & ND & + & S & ND & G2 & $\mathrm{P}[4]$ & A \\
\hline PGR08/005 & 2 & ND & + & - & - & NA & NA & NA \\
\hline PGR08/006 & 1 & ND & + & $\mathrm{S}$ & ND & NTD & $\mathrm{P}[4]$ & A \\
\hline PGR08/007 & 3 & ND & + & S & ND & NTD & $\mathrm{P}[4]$ & A \\
\hline PGR08/008 & 3 & ND & + & - & - & NA & NA & NA \\
\hline PGR08/009 & 10 & ND & + & - & - & NA & NA & NA \\
\hline PGR08/010 & 6 & ND & + & - & - & NA & NA & NA \\
\hline PGR08/011 & 1 & ND & + & S & ND & G2 & $\mathrm{P}[4]$ & A \\
\hline PGR08/012 & 6 & ND & + & - & - & NA & NA & NA \\
\hline PGR08/013 & 22 & ND & + & $\mathrm{S}$ & ND & G2 & $\mathrm{P}[4]$ & A \\
\hline PGR08/014 & 2 & ND & + & - & - & NA & NA & NA \\
\hline PGR08/015 & 2 & $N D$ & + & S & ND & NTD & NTD & A \\
\hline PGR08/016 & 1 & ND & + & $\mathrm{S}$ & ND & NTD & $\mathrm{P}[4]$ & A \\
\hline PGR08/017 & 52 & ND & + & S & ND & NTD & NTD & A \\
\hline PGR08/018 & 21 & ND & + & S & + & G2 & $\mathrm{P}[4]$ & A \\
\hline PGR08/019 & 3 & ND & + & - & - & NA & NA & NA \\
\hline PGR08/020 & 3 & ND & + & - & - & NA & NA & NA \\
\hline PGR08/021 & 4 & ND & + & S & ND & NTD & $\mathrm{P}[4]$ & A \\
\hline PGR08/022 & 5 & 10 & + & 5 & ND & G2 & $\mathrm{P}[4]$ & A \\
\hline PGR08/023 & 4 & ND & + & S & ND & NTD & $\mathrm{P}[4]$ & A \\
\hline PGR08/024 & 3 & 11 & + & S & ND & G2 & $\mathrm{P}[4]$ & A \\
\hline PGR08/025 & 30 & ND & + & S & ND & G2 & $\mathrm{P}[4]$ & A \\
\hline PGR08/026 & 7 & ND & + & - & - & NA & NA & NA \\
\hline PGR08/027 & 63 & ND & + & S & + & NTD & NTD & A \\
\hline PGR08/028 & 6 & $1 \mathrm{NI}$ & + & - & - & NA & NA & NA \\
\hline PGR08/029 & 45 & ND & + & S & ND & G2 & NTD & A \\
\hline PGR08/030 & 1 & ND & + & S & ND & G2 & $\mathrm{P}[4]$ & A \\
\hline PGR08/031 & 4 & ND & + & 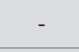 & - & NA & NA & NA \\
\hline PGR08/032 & 4 & ND & + & S & ND & G2 & $\mathrm{P}[4]$ & A \\
\hline PGR08/033 & 1 & ND & + & S & + & NTD & NTD & A \\
\hline PGR08/034 & 1 & ND & + & - & ND & NA & NA & NA \\
\hline
\end{tabular}

aEletropherotype: L - long, S - short.

bAntigenic specificity carried by indicated proteins.

${ }^{\mathrm{N} D}$, not done.

${ }^{\mathrm{d}} \mathrm{NA}$, not amplified.

eNTD, not determined.

+ positive/- negative. 
Eleven samples amplified, individually, either for NSP4-A (6) or NSP4-B (5). Thirteen samples amplified for the double combinations G1/NSP4-B (2), G1P[8] (1), G2/NSP4-A (1), P[4]/NSP4-A (6), and P[8]/NSP4-B (3). Twenty samples amplified for the triple combinations G1P[8]/NSP4-B (6), G2P[4]/NSP4-A (12) and G9P[8]/NSP4-B (2). Genotypes G1, G2 and G9 accounted for 37.5\% (9/24), 54.1\% (13/24), and $8.3 \%(2 / 24)$ of the strains detected, respectively. In our study genotyping VP4 demonstrated that $\mathrm{P}$ [4] was prevalent in $56.2 \%(18 / 32)$ in comparison to $43.7 \%(14 / 32)$ of $\mathrm{P}[8]$. Genotyping of NSP4 accounted for 56.8\% (25/44) for type A and $43.2 \%$ for type B.

According to the ages of the patients, out of 124 stool samples 57 came from children and young children ( $\leq$ twelve years of age) $(82.6 \%, 57 / 69)$. Positive cases in adults were detected in twelve patients over 12 years, including an 81-year-old male patient, overall representing $17.4 \%$ $(12 / 69)$ (Table 3$)$.

Table 3. Distribution of rotavirus positive cases according to the age of the patients

\begin{tabular}{|cc|}
\hline Age group (year) & n (\%) \\
\hline$\geq 2$ & $17(24.6)$ \\
\hline $2-12$ & $40(58.0)$ \\
\hline$>12$ & $12(17.4)$ \\
\hline Total & $69(100)$ \\
\hline
\end{tabular}

\section{DISCUSSION AND CONCLUSION}

This study demonstrated the outstanding importance of $\mathrm{RV}$ as the causative of acute diarrheal disease, accounting for a positivity of $55.6 \%$, particularly because all the subjects studied were hospitalized patients. Overwhelmingly, most of the RV positive stool samples were from children under six years of age. This epidemiological feature has been taken for granted, but, increasingly, adults have been affected by the disease. Infection of RV in adults seems to be common and has been reported mainly among geriatric patients, disabled individuals, health attendants and those living in households with diseased children. ${ }^{22-25}$ In the present work, we found that $17.4 \%$ of $\mathrm{RV}$ positive samples were detected in adults from 12 years of age, including an 81-year old patient. It is suggested that the number of adults infected by RV would be greater, and studies involving them should be encouraged. It has been suggested that symptomatic or asymptomatic adults could serve as source of infection to children. ${ }^{26,27}$ In this study RV-A, as a rule, accounted for $97.1 \%$ of the cases, as most of the world reports have demonstrated..$^{28-30}$ The electropherotypes of these strains of RV-A showed a slight predominance of short pattern in comparison to the long-pattern strains, albeit, unusual, this has been reported in India. ${ }^{31}$ In general, the predominance of long e-types has been reported throughout the world, ${ }^{32-36}$ however, variation has been shown according to some genotypes. As far as VP7 genotyping is concerned, our study revealed greater incidence of G2 secondly by G1. Although in general, greater incidence has been attributed to G1 worldwide, in Brazil, however, temporally/seasonally there has been a change of prevalence between G1 and G2, as demonstrated elsewhere..$^{29,37}$ In our study, the greater incidence of G2 may be explained by geographical features or due to the small sampling. Nevertheless, these results demonstrated the occurrence of $\mathrm{G}$ types common in most of the countries, including Brazil ${ }^{9,10,37}$ combined with $\mathrm{P}[4]$ or $\mathrm{P}[8]$. For VP4 genotyping, similar prevalence profile has been found in other countries and in Brazil, and the analysis underlying varied epidemiological features is also possible, between the two major genotypes presently found, P[4] and P[8]. As for NSP4 genotyping, we found that type A accounted for the most strains detected. B type NSP4 has been shown the most common in the world. ${ }^{11,12}$ However, in Brazil, this is true for the southeastern area, ${ }^{38}$ but not for the northern region, where A type is prevalent. ${ }^{39}$ The data reported in our work demonstrated similar profile of the wild circulating strains of $\mathrm{RV}$, as compared to data obtained elsewhere in the world and in our country. Molecular and serological nuances are mainly attributed to factors, such as RV genotypic and phenotypic variability; interspecies barriers crossing; host immunity pressure - naturally or artificially acquired, as well as, geographical and seasonal features. The apparent increase of adult infection and the increasing number of untyped strains, albeit, positive by other tests, as we found in our work, may be a demonstration of a constant genetic variability. Therefore, the emergence of new RV strains should be expected. The effect of RV vaccination recently launched in Brazil is of major benefit for preventing the disease. However, changing in RV genotypes might also be expected as a result of a selective evolutionary process. In concluding, the threat posed by RV still represents a heavy sanitary and economical burden and has to be carefully treated.

\section{ACKNOWLEDGMENTS}

This work is part of GFK M.Sc. manuscript and the authors wish to thank to $\mathrm{CNPq}$, Brazilian Ministry of Health, CAPES, Fundação Araucária and PROPPG/UEL for financial aids. 


\section{REFERENCES}

1. Angel J, Franco MA, Greenberg HB. Rotavirus vaccines: recent developments and future considerations. Nat Rev Microbiol 2007;5:529-39.

2. Estes MK, Cohen J. Rotavirus gene structure and function. Microbiological Reviews 1989;53:410-49.

3. Yeager M, Dryden KA, Olson NH et al. Three-dimensional structure of rhesus rotavirus by cryoelectron microscopy and image reconstruction. J Cell Biol 1990;110:2133-44.

4. Estes MK, Kapikian AZ. Rotaviruses. In: Knipe D.M., Howley PM, Griffin DE et al. eds. Fields Virology. Philadelphia: Kluwer Healt Lippincott, Williams \& Wilkins, 2007.

5. Iturriza-Gómara $\mathrm{M}$, Wong $\mathrm{C}$, Blome $\mathrm{S}$, Desselberger U, Gray J. Molecular characterization of VP6 genes of human rotavirus isolates: correlation of genogroups with subgroups and evidence of independent segregation. J Virol 2002;76:6596-601.

6. Abe M, Ito N, Morikawa S et al. Molecular epidemiology of rotaviruses health calves in Japan: Isolation of a novel bovine rotavirus bearing new $\mathrm{P}$ and $\mathrm{G}$ genotypes. Virus Res 2009;144:250-7.

7. Ursu K, Kisfali P, Rigó D et al. Molecular analysis of the VP7 gene of pheasant rotavirus identifies a new genotype designated G23. Arch Virol 2009;154:1365-9.

8. Matthijnssens J, Ciarlet M, Rahman M et al. Recommendations for the classification of group A rotaviruses using all 11 genomic RNA segments. Arch Virol 2007;153:1621-9.

9. Beards GM, Desselberger U, Flewett TH. Temporal and geographical distributions of human rotavirus serotypes, 1983 to 1988. J Clin Microbiol 1989;27:2827-33.

10. Santos N, Hoshino Y. Global distribution of rotavirus serotypes/genotypes and its implication for the development and implementation of an effective rotavirus vaccine. Rev Med Virol 2005;15:29-56.

11. Iturriza-Gómara M, Anderton E, Kang G et al. Evidence for genetic linkage between the gene segments encoding NSP4 and VP6 proteins in common and reassortant human rotavirus strains. J Clin Microbiol 2003;41:3566-73.

12. Cho SL, Ahn JH, Kim K et al. Genetic variation in the NSP4 gene of human rotavirus isolated in Seoul. J Bacteriol Virol 2006;36:79-87.

13. Espejo RT, Calderón E, González N et al. Presence of two distinct types of rotavirus in infants and young children hospitalized with acute gastroenteritis in Mexico City, 1977. J Infect Dis 1979;139:474-7.

14. Matsuno S, Hasegawa A, Mukoyama A, Inouye S. A candidate for a new serotype of human rotavirus. J Virol 1985;54:623-4.

15. Vesikari T, Matson DO, Dennehy P et al. Safety and efficacy of a pentavalent human-bovine (WC3) reassortant rotavirus vaccine. N Engl J Med 2006;354:23-33.

16. Ruiz-Palacios GM, Pérez-Schael I, Velázquez FR et al. Safety and efficacy of an attenuated vaccine against severe rotavirus gastroenteritis. N Engl J Med 2006;354:11-22.

17. Solberg OD, Hasing ME, Trueba G, Eisenberg JNS. Characterization of novel VP7, VP4, and VP6 genotypes of a previously untypeable group A rotavirus. Virology 2009;385:58-67.

18. Santos N, Nozawa C. Comparação dos testes de aglutinação do látex, microscopia eletrônica e eletroforese em gel de poliacrilamida do RNA viral na detecção de rotavírus em fezes diarréicas de crianças. Revista Brasileira de Patologia Clínica 1989;25:117-20.

19. Herring AJ, Inglis NF, Ojeh CK et al. Rapid diagnosis of rotavirus infection by direct detection of viral nucleic acid in silverstained polyacrylamide gels. J Clin Microbiol 1982;16:473-7.
20. Santos N, Soares CC, Volotão EM et al. Surveillance of rotavirus strains in Rio de Janeiro, Brazil, from 1997 to 1999. J Clin Microbiol 2003;41:3399-402

21. Santos N, Honma S, Timenetsky MCST et al. Development of a microtiter plate hybridization-based PCR-enzyme-linked immunosorbent assay for identification of clinically relevant human group A rotavirus G and P genotypes. J Clin Microbiol 2008;46:462-9.

22. Wenman WM, Hinde D, Feltham S, Gurwith M. Rotavirus infection in adults. Results of a prospective family study. N Engl J Med 1979;301:303-6.

23. Anderson EJ, Weber SG. Rotavirus infection in adults. Lancet Infect Dis 2004;4:91-9.

24. Iijima Y, Iwamoto T, Nukuzuma S et al. An outbreak of rotavirus infection among adults in an institution for rehabilitation: long-term residence in a closed community as a risk factor for rotavirus illness. Scand J Infect Dis 2006;38:490-6.

25. Carraro E, Perosa AHS, Siqueira I et al. Rotavirus infection in children and adult patients attending in a tertiary hospital of São Paulo, Brazil. Braz J Infect Dis 2008;12:44-6.

26. Barnes GL, Callaghan SL, Kirkwood CD et al. Excretion of serotype G1 rotavirus strains by asymptomatic staff: a possible source of nosocomial infection. J Pediatr 2003;142:722-5.

27. Pietruchinski E, Benati F, Lauretti F et al. Rotavirus diarrhea in children and adults in a southern city of Brazil in 2003: distribution of G/P types and finding of a rare G12 strain. J Med Virol 2006;78:1241-9.

28. Parashar UD, Gibson CJ, Bresse JS, Glass RI. Rotavirus and severe childhood diarrhea. Emerg Infect Dis 2006;12:304-6.

29. Munford V, Souza EC, Caruzo TAR et al. Serological and molecular diversity of human rotavirus in São Paulo, Brazil. Braz J Microbiol 2007;38:459-66.

30. Surendran S. Rotavirus infection: molecular changes and pathophysiology. Experimental and Clinical Sciences Journal 2008;7:154-62.

31. Saravanan P, Ananthan S, Ananthasubramanian M. Rotavirus infection among infants and young children in Chennai, South India. Indian J Med Microbiol 2004;22:212-21.

32. Maunula L, von Bonsdorff C-H. Frequent reassortments may explain the genetic heterogeneity of rotaviruses: analysis of Finnish rotavirus strains. J Virol 2002;76:11793-800.

33. Urbina D, Rodríguez JG, Arzuza O et al. G and P genotypes of rotavirus circulating among children with diarrhea in the Colombian northern coast. Int Microbiol 2004;7:113-20.

34. Modarres S, Rahbarimanesh AA, Karimi $\mathrm{M}$ et al. Electrophoretic RNA genomic profiles of rotavirus strains prevailing among hospitalized children with acute gastroenteritis in Tehran, Iran. Arch Iran Med 2008;11:526-31.

35. Aminu M, Esona MD, Geyer A, Steele AD. Epidemiology of rotavirus and astrovirus infections in children in northwestern Nigeria. Ann Afr Med 2008;7:168-74.

36. Santos JS, Alfieri AF, Leite JPG et al. Molecular epidemiology of the human group a rotavirus in the Paraná State, Brazil. Brazilian Archives of Biology and Technology 2008;51:287-94.

37. Leite JP, Carvalho-Costa FA, Linhares AC. Group A rotavirus genotypes and the ongoing Brazilian experience: a review. Mem Inst Oswaldo Cruz 2008;103:745-53.

38. Araújo IT, Heinemann MB, Mascarenhas JDP et al. Molecular analysis of NSP4 and VP6 genes of rotavirus strains recovered from hospitalized children in Rio de Janeiro, Brazil. J Med Microbiol 2007;56:854-9.

39. Mascarenhas JDP, Linhares AC, Gabbay YB et al. Molecular characterization of VP4 and NSP4 genes from rotavirus strains infecting neonates and young children in Belem, Brazil. Virus Res 2007;126:149-58. 